\title{
Effect of Feeding by Aromatic Honey Anise (Pimpinella anisum) \& Fennel (Foeniculum vulgar) and Marjoram (Origanum majorana) on Nosema Disease
}

\author{
Darwish, M. G. \\ Plant Prot. Dept. Faculty of Agriculture, Minia University
}

Received on: 21-10-2021

Accepted on: 23-11-2021

\begin{abstract}
Nosema disease is regarded one of the causes of Colony Collapse Disorder (CCD). Nowadays, aromatic honey has significant effect in honey-bee diseases and it is not unusual, feeding honey bees by aromatic honey for Nosema disease control. Results revealed that feeding honey bee on Anise honey by concentration $150 \mathrm{~g} / \mathrm{colony}$ have the lowest infection percentage $28.78 \%$ then Anise $100 \mathrm{~g} / \mathrm{colony} 32.94 \%$ and Anise $50 \mathrm{~g} / \mathrm{colony} 37.89 \%$ compared with control $65.11 \%$. In the second place Fennel honey general mean $43.11 \%$. Finally, Marjoram honey general mean $62.76 \%$.

On the other hand, the effect of these aromatic honey samples back to that antioxidant activity of the three types of honeys represented in flavonoids value. The chemical analysis of honey samples was carried out to clarify the beneficial compounds that have an effect on Nosema disease. Results showed that the highest flavonoids value was found in Anise honey (14.02) followed by Fennel honey (9.11) and finally Marjoram honey (8.24).
\end{abstract}

KEYWORDS: Honeybee - nosema - aromatic

\section{INTRODUCTION}

Nosema disease is a parasitic disease infects adult bees of Apis mellifera L. (Milbrath et al., 2015) and belonging to fungal honeybee diseases (Moshaverinia et al., 2012). This disease causes great losses in honey bee colonies around the world (Jara et al., 2015 and Roussel et al., 2015). Also, Bromenshenk et al., 2010 revealed that Nosema disease was considered the most important reasons of CCD, Moreover, Malone et al., 2001 cleared that spores of Nosema lasted viability after feeding bees on multifloral honey. The most plants that have potential feeding importance to honey bee are belonging to medicinal, aromatic and ornamental plants with $35.2 \%$ of total plants Aboushaara 2015. Bees collect nectar that secreted by glands at the base of the flowers. The nectar has sucrose sugar with some laevulose, dextrose, moisture content and aromatic substances which have big role in microorganism control. Furthermore, honey combs can be stored and given to colonies as required (Doug 2000). Aromatic compounds have a big role against microorganisms causing diseases to humans, animals and plants in addition to its considered that safely used as an alternative to pesticides (Altundağ \& Aslım, 2005). Also, Mert et al, 2007 showed that Nosema disease control depending on using aromatic compounds as well as other honeybee diseases and enemies such as Varroa mite, tracheal mite American foulbrood, European foulbrood and wax moths.

\section{MATERIALS AND METHODS}

\subsection{Honey preparation:}

Anise (Pimpinella anisum), Fennel (Foeniculum vulgar) and Marjoram (Origanum majorana) honey syrup:

Three concretions $50 \mathrm{~g}, 100 \mathrm{~g}$ and $150 \mathrm{~g}$ from each of Anise, Fennel and Marjoram honey for each colony. Each concentration was applied in $250 \mathrm{ml}$ water weekly. Control colonies fed by sugar syrup ( 2 sugar: 1 water, w/v).

\subsection{Experimental bees:}

The present study was carried out from November 2019 to April 2020. Twenty-seven of hybrid carniolan honeybee colonies (Apis mellifera) were placed on Minia region. Samples of 100 adult honeybee workers were collected randomly from the front of entrances of the hives (Shimanuki \& Knox, 2000; Vongpakorn \& Neramitmansook, 2003 and Matasin et al., 2012). Samples were taken monthly 


\section{Darwish, M. G., 2021}

and adult bees were crushed individually with distilled water in a mortar (Topolska \& Hartwig, 2005 \& Lotfi et al., 2009). After preparation on a glass slide, each sample was examined by a light microscope at $\mathrm{x} 400$ magnification for the presence of Nosema spores (Razmaraii et al., 2013). The percentage of infected bees was determined according to the following equation (Topolska, \& Hartwig, 2005).

No. of infected bees

Infection $\%=100 \mathrm{x}$

Total no. of bees

Data were statistically analyzed by using least signific ant range (Duncan, 1955). Chemical analysis w as carried out according to Kaspar, et al. 2006.

\section{RESULT AND DISCUSSION}

Data in Table (1) and Figure (1 and 2) showed that the mean efficiency of the tested aromatic honeys in reducing Nosema infection could be arranged in the following descending order; Anise 150g > Anise 100g $>$ Anise $50 \mathrm{~g}>$ Fennel $150 \mathrm{~g}>$ Fennel $100 \mathrm{~g}>$ Fennel $50 \mathrm{~g}>$ Marjoram 100g > Marjoram 150g > Marjoram $50 \mathrm{~g}>$ control resulted in $28.78 \%, 32.94 \%, 37.89 \%$, $38.11 \%, 42.39 \%, 48.83 \%, 62.28 \%, 62.39 \%, 63.61 \%$ and $65.11 \%$, respectively. Concluded from this data feeding by Anise with concentration $150 \mathrm{~g}$ honey showed the lowest infection percent $(28.78 \%)$ by Nosema compared with the other treatments.

By other words, results in Fig. (2 and 3) showed that using Anise honey helped in minimizing Nosema general mean infection $33.20 \%$ then Fennel honey $43.11 \%$ finally Marjoram honey $62.76 \%$.

Also, Statistical analysis showed significant different between feeding by from Anise, Fennel and Marjoram honey because most means followed by different letters. These data agreement with Gashout \& Guzmán-Novoa 2009 and Lin et al., 2020.

Table 1. Monthly Nosema infection percentage of honeybee colonies which fed Marjoram honey (50g \& $100 \mathrm{~g}$ and $150 \mathrm{~g} /$ colony) from November 2019 to April 2020

\begin{tabular}{|c|c|c|c|c|c|c|c|c|c|c|}
\hline & \multicolumn{3}{|l|}{ Anise } & \multicolumn{3}{|c|}{ Fennel } & \multicolumn{3}{|c|}{ Marjoram } & \multirow[t]{2}{*}{ Control } \\
\hline & $50 \mathrm{~g}$ & $100 \mathrm{~g}$ & $150 \mathrm{~g}$ & $50 \mathrm{~g}$ & $100 \mathrm{~g}$ & $150 \mathrm{~g}$ & $50 \mathrm{~g}$ & $100 \mathrm{~g}$ & $150 \mathrm{~g}$ & \\
\hline November & $\begin{array}{l}37.67 \\
\text { Opq }\end{array}$ & $\begin{array}{l}\text { 37.00 } \\
\text { pqr }\end{array}$ & $\begin{array}{l}38.00 \\
\text { nopq }\end{array}$ & $\begin{array}{l}\text { 37.00 } \\
\text { pqr }\end{array}$ & $\begin{array}{l}\text { 37.33 } \\
\text { pqr }\end{array}$ & $\begin{array}{l}36.67 \\
\text { qrs }\end{array}$ & $\begin{array}{l}37.67 \\
\text { opq }\end{array}$ & $\begin{array}{l}38.67 \\
\text { nopq }\end{array}$ & $\begin{array}{l}38.33 \\
\text { nopq }\end{array}$ & $\begin{array}{l}37.33 \\
\text { pqr }\end{array}$ \\
\hline December & $\begin{array}{l}31.33 \\
u\end{array}$ & $\begin{array}{l}26.33 \\
\text { wx }\end{array}$ & $\begin{array}{l}20.67 \\
y\end{array}$ & $\begin{array}{l}44.33 \\
\mathrm{ij}\end{array}$ & $\begin{array}{l}35.00 \\
\text { rs }\end{array}$ & $\begin{array}{l}31.67 \\
\mathrm{U}\end{array}$ & $\begin{array}{l}58.33 \\
\text { f }\end{array}$ & $\begin{array}{l}54.00 \\
\text { h }\end{array}$ & $\begin{array}{l}\mathbf{5 5 . 6 7} \\
\text { gh }\end{array}$ & $\begin{array}{l}59.00 \\
\text { f }\end{array}$ \\
\hline January & $\begin{array}{l}40.33 \\
\operatorname{lmn}\end{array}$ & $\begin{array}{l}36.67 \\
\text { qrs }\end{array}$ & $\begin{array}{l}31.00 \\
\text { uv }\end{array}$ & $\begin{array}{l}\mathbf{5 4 . 6 7} \\
\text { gh }\end{array}$ & $\begin{array}{l}43.67 \\
j k\end{array}$ & $\begin{array}{l}43.67 \\
\text { Jk }\end{array}$ & $\begin{array}{l}81.33 \\
b\end{array}$ & $\begin{array}{l}81.67 \\
\text { ab }\end{array}$ & $\begin{array}{l}80.67 \\
\text { b }\end{array}$ & $\begin{array}{l}82.33 \\
\text { ab }\end{array}$ \\
\hline February & $\begin{array}{l}32.33 \\
\text { tu }\end{array}$ & $\begin{array}{l}28.67 \\
\text { vw }\end{array}$ & $\begin{array}{l}25.67 \\
x\end{array}$ & $\begin{array}{l}\text { 63.00 } \\
\text { e }\end{array}$ & $\begin{array}{l}41.33 \\
\mathrm{klm}\end{array}$ & $\begin{array}{l}37.33 \\
\text { pqr }\end{array}$ & $\begin{array}{l}81.33 \\
\text { b }\end{array}$ & $\begin{array}{l}81.33 \\
\text { b }\end{array}$ & $\begin{array}{l}81.33 \\
b\end{array}$ & $\begin{array}{l}84.00 \\
\text { a }\end{array}$ \\
\hline March & $\begin{array}{l}46.33 \\
i\end{array}$ & $\begin{array}{l}36.67 \\
\text { qrs }\end{array}$ & $\begin{array}{l}34.33 \\
\text { st }\end{array}$ & $\begin{array}{l}54.00 \\
\text { h }\end{array}$ & $\begin{array}{l}54.33 \\
h\end{array}$ & $\begin{array}{l}44.33 \\
\mathbf{I j}\end{array}$ & $\begin{array}{l}68.00 \\
\text { d }\end{array}$ & $\begin{array}{l}63.67 \\
e^{6}\end{array}$ & $\begin{array}{l}65.00 \\
e^{6}\end{array}$ & $\begin{array}{l}71.00 \\
c\end{array}$ \\
\hline April & $\begin{array}{l}39.33 \\
\text { mnop }\end{array}$ & $\begin{array}{l}32.33 \\
\text { tu }\end{array}$ & $\begin{array}{l}23.00 \\
y\end{array}$ & $\begin{array}{l}40.00 \\
\text { mno }\end{array}$ & $\begin{array}{l}42.67 \\
\text { jkl }\end{array}$ & $\begin{array}{l}35.00 \\
\text { Rs }\end{array}$ & $\begin{array}{l}55.00 \\
\text { gh }\end{array}$ & $\begin{array}{l}54.33 \\
\text { h }\end{array}$ & $\begin{array}{l}53.33 \\
\text { h }\end{array}$ & $\begin{array}{l}57.00 \\
\text { fg }\end{array}$ \\
\hline Mean & 37.89 & 32.94 & 28.78 & 48.83 & 42.39 & 38.11 & 63.61 & 62.28 & 62.39 & 65.11 \\
\hline $\begin{array}{l}\text { General } \\
\text { mean }\end{array}$ & 33.20 & & & 43.11 & & & 62.76 & & & \\
\hline
\end{tabular}

For each row, means followed by the same letters are not significantly different at 5\% level of probability (Duncan multiple range test)

*L.S.R. Least significant range (Duncan range at 5\%). 
Scientific Journal of Agricultural Sciences 3 (2): 230-235, 2021

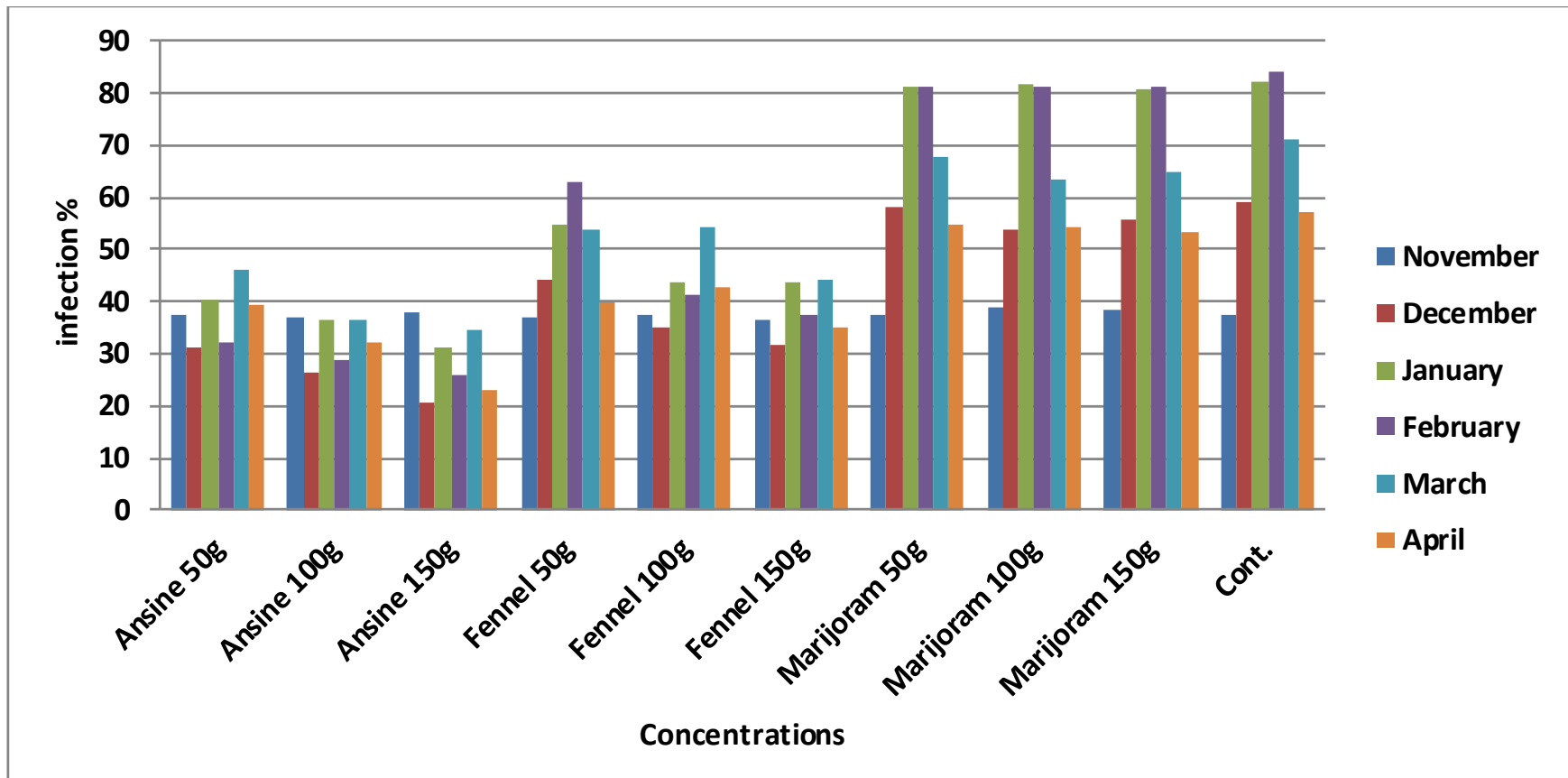

Figure 1. Mean monthly Nosema infection percentage of honeybee colonies which fed different concentrations of aromatic honey $(50 \mathrm{~g} \& 100 \mathrm{~g}$ and $150 \mathrm{~g} /$ colony) allover study period from November 2019 to April 2020.

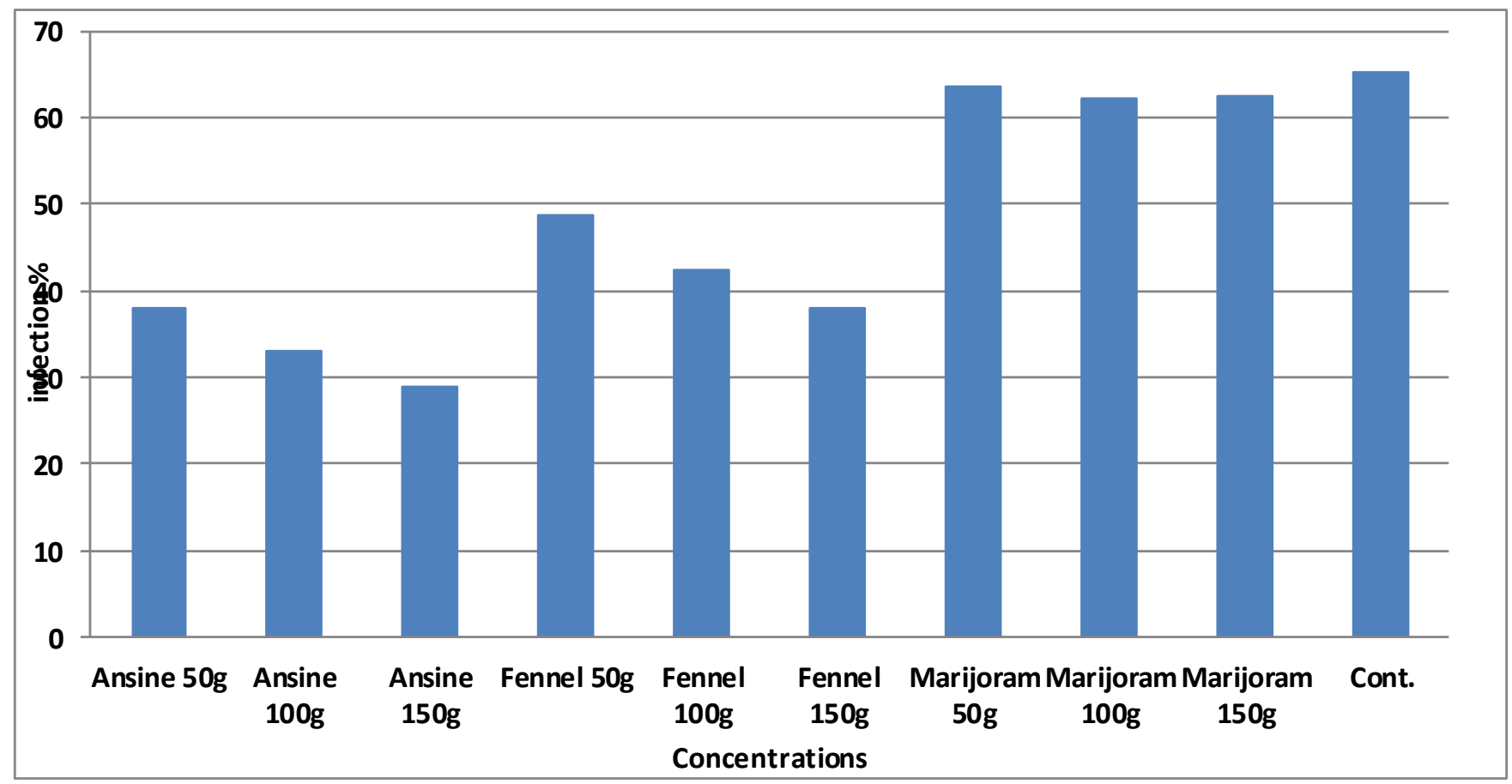

Figure 2. Mean Nosema infection percentage of honeybee colonies which fed by different aromatic honey from November 2019 to April 2020. 
Darwish, M. G., 2021

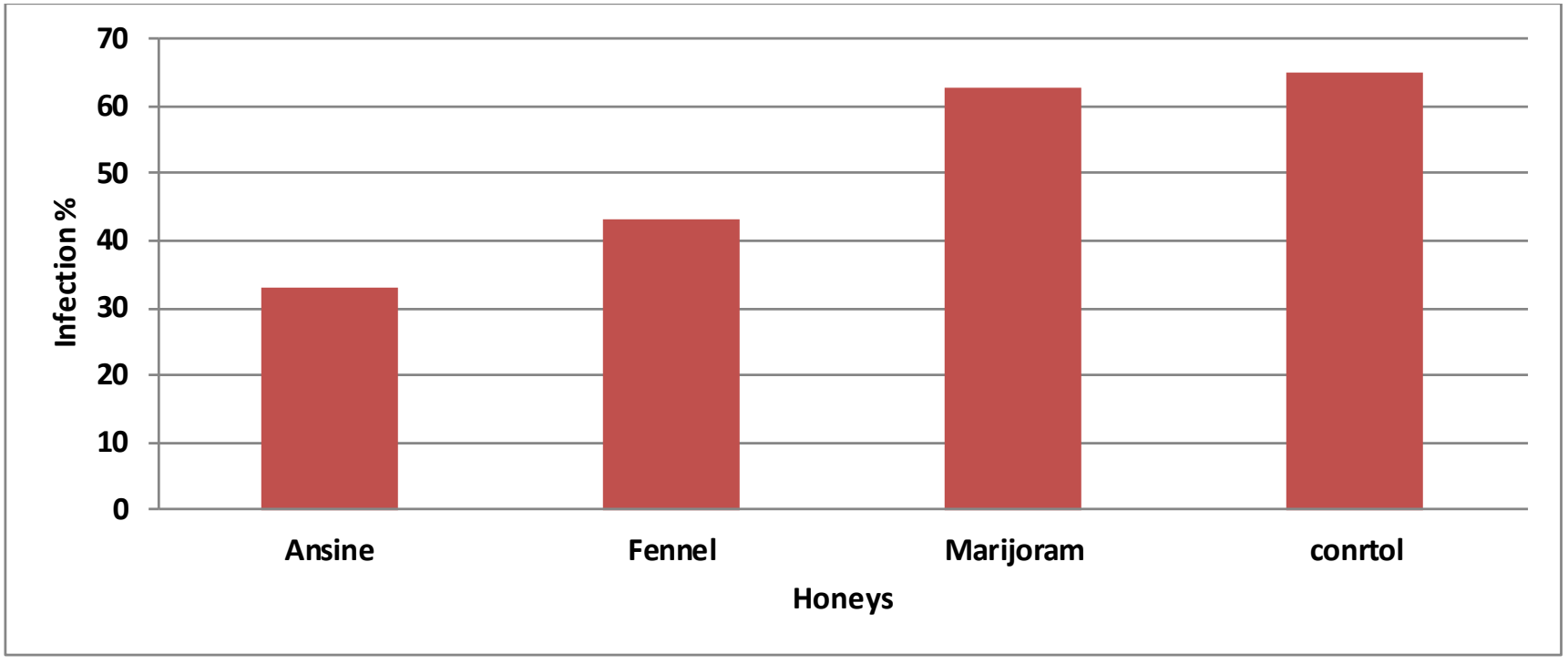

Figure 3. General mean of Nosema infection percentage of honeybee colonies which fed by different aromatic honey from November 2019 to April 2020.

Chemical analysis of teted honeys presented in table (2) and Figure (4) showed that Anise honey record the lowest concentration of TSS, glucose, sucrose, HMF and $\mathrm{PH}(79.56,34.01,1.36,12.26$ and 3.3, respectively) additionally, chemical analysis proved that the samples conform to the Codex Alimntarius (1998), Saudi standard (1990)' Gulf standard (1992) and the Egyptian standard (1990).
Also, data in table (2) and Figure (3) pointed that antioxidant activity of the three types of honeys represented in flavonoids was found higher in Anise honey (14.02) followed by, Fennel honey (9.11) and finally Marjoram honey (8.24). these results are in accordance with that reported by Kaspar, et al. 2006.

Table 2. Chemical analysis of Anise, Fennel and Marjoram honeys

\begin{tabular}{lccc}
\hline \multicolumn{1}{c}{ Samples } & $\begin{array}{c}\text { Anise } \\
\text { honey }\end{array}$ & $\begin{array}{c}\text { Fennel } \\
\text { honey }\end{array}$ & $\begin{array}{c}\text { Marjoram } \\
\text { honey }\end{array}$ \\
\hline TSS (\%) & & $\mathbf{8 1 . 8 5}$ & $\mathbf{8 0 . 1 9}$ \\
Moisture & $\mathbf{7 9 . 5 6}$ & $\mathbf{8 . 1 5}$ & $\mathbf{9 . 9 1}$ \\
Glucose (\%) & 10.44 & 35.23 & $\mathbf{3 4 . 6 3}$ \\
Sucrose (\%) & $\mathbf{3 4 . 0 1}$ & $\mathbf{2 . 8 6}$ & $\mathbf{2 . 2 2}$ \\
Fructose (\%) & 1.36 & $\mathbf{3 4 . 5 3}$ & $\mathbf{3 5 . 5 1}$ \\
HMF (mg/kg) & $\mathbf{3 8 . 3 6}$ & $\mathbf{1 8 . 4 3}$ & $\mathbf{2 1 . 0 0}$ \\
PH & 12.26 & $\mathbf{3 . 6 8}$ & $\mathbf{4 . 5 8}$ \\
Total Flavonoids & $\mathbf{3 . 3}$ & $\mathbf{9 . 1 1}$ & $\mathbf{8 . 2 4}$ \\
\hline
\end{tabular}


Scientific Journal of Agricultural Sciences 3 (2): 230-235, 2021

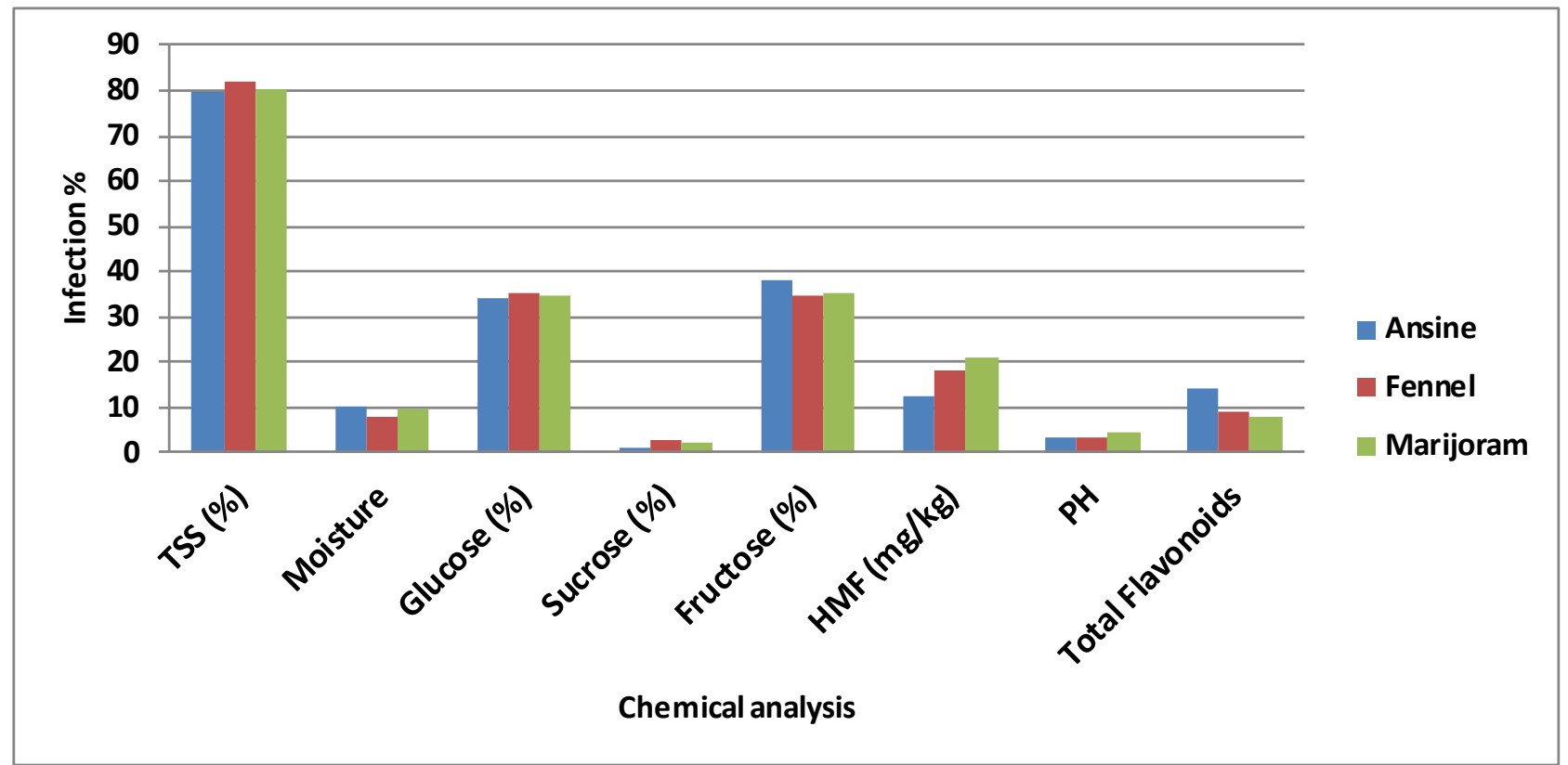

Figure 4. Chemical analysis of tested honeys Anise, Fennel and Marjoram

\section{REFERENCES}

Abou-shaara HF (2015). Potential Honey Bee Plants of Egypt. Cercetări Agronomice în Moldova Vol. XLVIII, No. 2 (162).

Altundag S, Aslim B (2005). Antimic robial effect of thyme on some plant pathogenic bacteria. Orlab OnLine Journal of Microbiology , 3 (7), 12-14.

Bromenshenk JJ, Bromenshenk JJ, Henderson CB, Wick CH, Stanford MF, Zulich AW, Jabbour, RE, Cramer Jr RA (2010). Iridovirus and microsporidian linked to honey bee colony decline. PloS one, 5(10), e13181.

Codex Alimntarius (1998). Draft revised for honey, at step 6 of the Codex Procedure. CX_5/10.2, CL 1998/ 12-S

Doug S (2000). Honey bee nutrition and supplementary feeding. State of New South Wales NSW Agriculture. (178).

Duncan DB (1955). Multiple Range and Multiple F Tests. Biometrics, 11(1), 1-42.

Egyptian standard (1990). Honey and Methods of test and experiment: chapter one: Honey. Egyptian standard (1990-355). Egyptian Organization for Standardization unification and production quality, A.R.E. 3 pp.

Gashout HA, Guzmán-Novoa E (2009). Acute toxicity of essential oils and other natural compounds to the parasitic mite, Varroa destructor and to larval and adult worker honey bees (Apis mellifera L.). Journal of apicultural research, 48(4), 263-269.

Gulf standard (1992). Review for Gulf standards consuming honey. Papers presented at technical seminar on honey standards, listing procedures and quality' control, Standardization and Metrology organization for the Gull' cooperation council countries and the United states national honey board. Riyadh. Saudi Arabia, p. 1-10.

Jara L, Munoz I, Cepero A, Martin-Hernandez R, Serrano J, Higes M, Rua P (2015). Stable genetic diversity despite parasite and pathogen spread in honeybee colonies. The Science of Nature. 102(9/10):53- 83

Kaspar R, Maria T, Werner L, Jacques-Olivier B, Stefan B, Renato A (2006). Quantitative analysis of physical and chemical measurands in honey by midinfrared spectrometry. Eur Food Res Technol. 223: 22-29

Lin Z, Su X, Wang S, JiT, Hu FL, Zheng HQ (2020) .Fumigant toxicity of eleven Chinese herbal essential oils against an ectoparasitic mite (Varroa destructor) of the honey bee (Apis mellifera). Journal of Apicultural Research, 59(2), 204-210.

Lotfi A, Jamshidi R, Shahryar HA, Yousefkhani M (2009). The prevalence of Nosemosis in honeybee colonies in Arasbaran region (northwestern Iran)._Am. Eurasian J. Agri. and Environ. Sci. 5(2):255-257.

Malone LA, Gatehouse HS, Tregidga EL (2001). Effects of time, temperature, and honey on Nosema apis (Microsporidia: Nosematidae), a Parasite of the Honeybee، Apis mellifera (Hymenoptera: Apidae). Journal of Invertebrate Pathology 77(4), 258-268.

Matasin Z, Nejedli S, Tlak Gajger I (2012). Leucine aminopeptidase activity in the midgut of nosema diseased honeybees (Apis mellifera). Veterinarski Arhiv. 82(6):599-607. 


\section{Darwish, M. G., 2021}

Mert G, Yücel B, Kösoğlu M (2007). Bal Arıs1 Hastalik ve Zararlları İle Organik Mücadele Yöntemleri, Hasad Hayvancılik, 261,263, 62-64, 5258.

Milbrath M, Toan V, Huang W, Solter L, Tarpy D, Lawrence F, Huang Z (2015). Comparative virulence and competition between Nosema apis and Nosema cerana in honeybees (Apis mellifera). J. Invert. Path. 125:9-15.

Moshaverinia A, Abedi V, Safaei H (2012). A survey of Nosema apis infection in apiaries of North Khorasan province, Iran. Iranian J. of Veterinary Sci. and Technology. 4(2):25-30.

Razmaraii N, Sadegh-Eteghad S, Babaei H, Paykari H, Esmaeilnia K, Froghy L (2013). Molecular identification of nosema species in East Azerbaijan province, Iran. Arch. of Razi Institute. 68(1):23-27.
Roussel M, Villay A, Delbac F, Michaud P, Laroche C, Roriz D, El-Alaoui H, Diogon M (2015). Antimicrosporidian activity of sulphated polysaccharides from algae and their potential to control honeybee nosemosis. Carbohydrate Polymers. 133:213-220.

Saudi standard (1990). Honey. Saudi Arabia organization for standardization and metrology, Saudi standard

Shimanuki H, Knox DA (2000). Diagnosis of honeybee diseases, US Depert. Agric., Agric. Hand. No. AH-690, pp. 61.

Topolska G, Hartwig A (2005). Diagnosis of Nosema apis infection by investigation of two kinds of samples. Api. Sci. 49(2):75-79.

Vongpakorn M, Neramitmansook W (2003). A survey of parasitic diseases in honeybee in Thailand. [Thai] Source Journal of the Thai Veterinary Medical Association. 54(3):19-27.

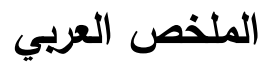

تأثير التغذية بالاعسال العطرية (اليانسون، الثمر والبردقوش) علي مرض النوزيما

محمود جمعة جمعة درويش

قسم وقاية النبات - كلية الزراعة - جامعة المنيا

$$
\begin{aligned}
& \text { يعتبر مرض النوزيما أحد أسباب انهيار طوائف نحل العل .في الوقت الحاضر ، ووجد أن لعسل النباتات العطرية تأثير كبير في مكافحة أمراض }
\end{aligned}
$$

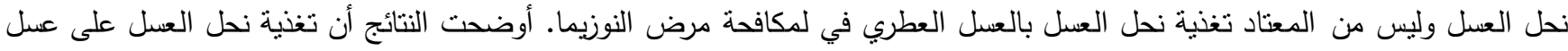

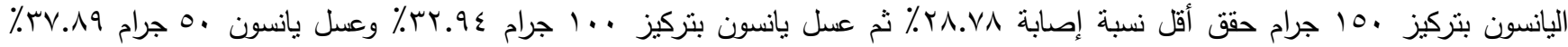

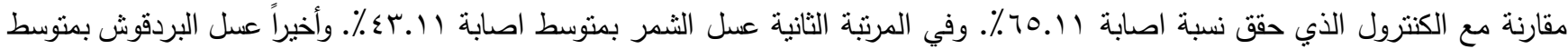

$$
\begin{aligned}
& \text { اصابة צV.VT\% } \\
& \text { من ناحية أخرى يعود ثأثثر العسل العطري إلى نثاط مضادات الأكسدة لأنواع العسل الثلاثة المتمثلة في مركبات الفلافونويد. نم إجراء التحليل }
\end{aligned}
$$

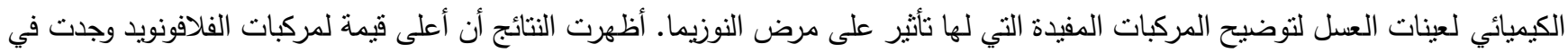

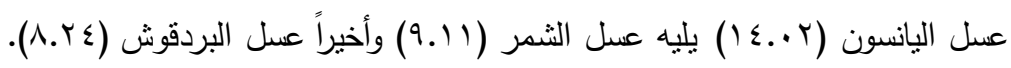

$$
\begin{aligned}
& \text { الكلمات المفتاحية: نحل العسل -نوزيما - عطري }
\end{aligned}
$$

